DOI: $10.15593 / 2224-9982 / 2016.47 .06$

УДК 629.7.036.54

\author{
А.И. Коломенцев ${ }^{1}$, А.Н. Хохлов ${ }^{2}$ \\ ${ }^{1}$ Московский авиационный институт \\ (национальный исследовательский университет), Москва, Россия \\ ${ }^{2}$ Центральный научно-исследовательский институт машиностроения, \\ Королев, Россия \\ ОПТИМАЛЬНОЕ ПЛАНИРОВАНИЕ ИСПЫТАНИЙ \\ ЖИДКОСТНЫХ РАКЕТНЫХ ДВИГАТЕЛЕЙ МАЛЫХ ТЯГ \\ ДЛЯ ОПРЕДЕЛЕНИЯ ИХ ОСНОВНЫХ ПАРАМЕТРОВ \\ И ХАРАКТЕРИСТИК
}

\begin{abstract}
Посвящено решению проблемы сокращения количества испытаний жидкостных ракетных двигателей малой тяги (ЖРДМТ). На сегодняшний момент появилась необходимость в разработке ЖРДМТ на экологически безопасных компонентах топлива (КТ), так как все новые проекты перспективных средств выведения и разгонных блоков планируются на высокоэнергетических экологически чистых и безопасных КТ - таких, как кислород + водород, кислород + метан, кислород + керосин, а следовательно, и реактивные системы управления должны быть на основных бортовых КТ. ЖРДМТ на таких КТ не имеют достаточного объема статистических данных по отработке конструкций.

Экспериментальная отработка ЖРДМТ является очень энергоемким и дорогим процессом. Чтобы сократить количество, а соответственно, стоимость испытаний, применяется планирование эксперимента. Существующие методики планирования и анализа результатов испытаний относятся, в основном, к ЖРД большой мощности. В статье рассмотрены различные планы экспериментов, проведен их анализ и сделан вывод об использовании оптимальных планов испытаний ЖРДМТ. На основании проведенных исследований двигателей тягой от 10 до $500 \mathrm{H}$ представлены общие виды регрессионных моделей и результирующие таблицы для них. Также результаты исследований приведены в графическом виде.

Ключевые слова: жидкостный ракетный двигатель малой тяги, планирование эксперимента, регрессионные модели, оптимальный план эксперимента.
\end{abstract}




\author{
A.I. Kolomentsev ${ }^{1}$, A.N. Khokhlov ${ }^{2}$ \\ ${ }^{1}$ Moscow Aviation Institute (National Research University), \\ Moscow, Russian Federation \\ ${ }^{2}$ Central Research Institute of Machine Building, Korolev, Russian Federation

\section{OPTIMAL PLANNING TESTS OF LIQUID-PROPELLANT THRUSTERS FOR DETERMINATION OF THEIR GENERAL PARAMETERS AND CHARACTERISTICS}

Article is devoted to solution of the problem of test number reduction for the liquid-propellant thrusters (LPT). At present, there is a need to develop LPT on pollution-free propellant components, because all new projects of perspective launch vehicles are planned to be on safety pollution-free highpower propellant components, such as oxygen + hydrogen, oxygen + methane, oxygen + kerosene. Thus, reactive control systems should be operated on the basic onboard propellant components. LPT with such propellant components have no enough statistical data on static tests.

Static tests of LPT is very power-intensive and expensive process. To reduce quantity and respectively a cost of tests the planning an experiment is applied. The existing techniques of planning an experiment and analysis of test results are generally applicable to liquid rocket engines with high levels of thrust. The article considers different plans of experiments; their analysis is carried out. The conclusion is drawn on use of optimum plans of LTP tests. Overview of regression models and the resulting tables for them are provided based on conducted research of engines with thrust from 10 to $500 \mathrm{~N}$. The results are also given in graphical form.

Keywords: liquid-propellant thruster, planning an experiment, regression models, optimal design of the experiment.

Испытания ЖРДМТ имеют несколько особенностей:

1) кратковременность;

2) повышенную опасность;

3) высокую стоимость;

4) разнообразие видов испытаний и методов их проведения.

Стоимость испытаний связана в первую очередь с уникальностью испытательного оборудования. В связи с этим возникают требования высокой информативности испытаний и рационального их планирования, позволяющие получить более полные данные о двигателе в кратчайшее время.

Снижение стоимости отработки ракетных двигателей может быть достигнуто за счет применения научных методов планирования испытаний и обработки экспериментальных данных. При этом широко используются методы моделирования, математической статистики, факторного анализа и теории надежности $[1,2]$. 
Планирование эксперимента - это процедура выбора числа и условий проведения опытов, необходимых и достаточных для решения поставленной задачи с требуемой точностью. При этом существенно следующее:

1) стремление к минимизации общего числа испытаний;

2) одновременное варьирование всех переменных, определяющих процесс, по специальным правилам - алгоритмам;

3) выбор четкой стратегии, позволяющей принимать обоснованные решения после каждой серии опытов.

При планировании эксперимента очень важно определить параметр, который нужно оптимизировать [2-4]. Планирование начинается с выбора области испытаний (эксперимента). В первом приближении область определения факторов (т.е. совокупность всех значений существенных факторов) может быть задана с учетом допусков на входные факторы, указанных в техническом задании (Т3) на проектирование двигателя. Однако часто диапазон допусков невелик и требуется большое число экспериментов (для ЖРД более 40) для достижения приемлемой статистической точности. В связи с этим область определения факторов желательно расширить за пределы допусков, но с учетом ограничений, связанных прежде всего с работоспособностью двигателя, а также имея в виду то, что с увеличением интервалов варьирования факторов может потребоваться усложнение вида модели.

Параметр оптимизации - это признак, по которому оптимизируется процесс.

Фактором называется измеряемая переменная величина, принимающая в некоторый момент времени определенное значение [3, 5]. При планировании эксперимента факторы должны быть управляемыми. Точность замера факторов должна быть возможно более высокой. Степень точности определяется диапазоном изменения факторов (желательно, чтобы ошибка измерений составляла не более 2 \%). Факторы должны быть однозначны и оказывать непосредственное воздействие на объект.

При планировании эксперимента обычно одновременно изменяются несколько факторов, поэтому очень важно сформулировать следующие требования, которые предъявляются к совокупности факторов.

Область эксперимента задается уровнями и интервалами варьирования факторов. Интервал варьирования $\Delta x_{i}$ - это расстояние на 
оси $x_{i}$ между основным и верхним $\left(x_{i}\right)_{\max }$ уровнями факторов. Минимальная величина интервала варьирования должна быть больше ошибок воспроизведения уровней факторов в эксперименте, иначе верхний и нижний уровни будут неразличимы. При одинаковых ошибках эксперимента с увеличением интервала варьирования коэффициенты регрессии оцениваются точнее.

Для упрощения записи условий эксперимента и обработки экспериментальных данных значения факторов приводят к безразмерному виду:

$$
\bar{x}_{i}=\frac{x_{i}-\left(x_{i}\right)_{\text {ном }}}{\Delta x_{i}}
$$

где $\left(x_{i}\right)_{\text {ном }}$ - значение $i$-го входного фактора на номинальном режиме. Тогда верхний уровень равен +1 , нижний -1 , а основной - нулю.

Пусть имеется $k$-мерное пространство и $n$ измерений выходного параметра в некоторых точках этого пространства. Каждая точка представляет собой вектор $\left\{x_{i j}\right\}$, где $i=1, \ldots, k ; j=1, \ldots, n$. Набор этих точек по всем $n$ опытам называется планом эксперимента. План должен отвечать следующим основным требованиям:

1) минимум числа опытов;

2) композиционность;

3) ортогональность;

4) ротатабельность.

Выбор модели - это выбор вида функции (записи уравнения) регрессии. На основании физического анализа рабочих процессов, протекающих в двигателе, и с учетом ранее накопленного опыта выдвигается гипотеза о форме связи между выходным параметром $Y$ и входными факторами $x_{i}$, т.е. о структуре регрессионной модели. Основные требования к модели - это адекватность и простота.

Очень часто математическое ожидание выходного параметра представляют в виде полинома

$$
E(Y)=\beta_{0}+\sum_{i=1}^{k} \beta_{i} x_{i}+\sum_{\substack{i, j=1 \\ i<j}}^{k} \beta_{i j} x_{i} x_{j}+\sum_{i=1}^{k} \beta_{i i} x_{i}^{2}+\ldots
$$


где $\beta$ - коэффициенты регрессии; $\beta_{0}$ равно значению выходного параметра на номинальном режиме работы двигателя; $\beta_{i}$ равно тангенсу угла наклона касательной к поверхности функции отклика по отношению к оси $i$-го фактора.

Полиномом можно описать, с наперед заданной точностью, любую функцию, непрерывную в какой-либо ограниченной области факторного пространства. Степень полинома должна выбираться возможно меньшей, так как чем она ниже, тем меньше в уравнении коэффициентов регрессии и, следовательно, меньше требуется опытов для их определения; также это необходимо для упрощения систем обработки уравнений, например в системах управления или аварийной защиты.

Простейшим видом модели такого типа является линейная регрессионная модель

$$
E(Y)=\beta_{0}+\beta_{1} x_{1}+\beta_{2} x_{2}+\ldots+\beta_{k} x_{k} .
$$

Линейная модель будет адекватной при правильном выборе подобласти в факторном пространстве.

Используются также и другие виды регрессионной модели:

- степенная

$$
E(Y)=\beta_{0} x_{1}^{\beta_{1}} x_{2}^{\beta_{2}} \ldots x_{k}^{\beta_{k}} ;
$$

- экспоненциальная

$$
E(Y)=\beta_{0} \beta_{1}^{x_{1}} \beta_{2}^{x_{2}} \ldots \beta_{k}^{x_{k}}
$$

- комбинированные.

Существует большое количество планов для построения различных моделей: полный факторный эксперимент, дробный факторный эксперимент, центральный композиционный план, план Хартли, план Бокса-Вилсона, планы Бокса-Бенкена и др. [6-10]. Целью исследования является выбор наиболее подходящего плана для испытаний ЖРДМТ.

В ЖРД принято использовать полный факторный, дробный факторный, центральный композиционный («звездный») планы и малый центральный композиционный план (план Хартли) [9, 11].

Для ЖРДМТ основными выходными параметрами являются: тяга $P=\dot{m} W_{a}+F_{a}\left(p_{a}-p_{H}\right)$, удельный импульс тяги $I_{y}=\frac{P}{\dot{m}}=$ 
$=W_{a}+\frac{F_{a}}{\dot{m}}\left(p_{a}-p_{\mathrm{H}}\right)=W_{\text {эф }}$, импульс тяги $I_{\Sigma}=\int_{0}^{\tau_{\text {раб }}} P(\tau) d \tau$ и тепловое состояние (температура конструкции двигателя) [12-14].

В качестве выходных параметров выбраны удельный импульс тяги, так как он показывает энергетическую эффективность работы двигателя, и температура внешней стенки камеры двигателя, показывающая тепловое состояние двигателя.

Для удельного импульса управляемыми факторами являются:

1) $\dot{m}_{\text {о }}$ - массовый расход окислителя, кг/с;

2) $\dot{m}_{\Gamma}-$ массовый расход горючего, кг/с;

3) $T_{\text {o }}$ - температура окислителя на входе в двигатель, К;

4) $T_{\text {г }}$ - температура горючего на входе в двигатель, К;

5) $p_{\text {к }}$ - давление в камере сгорания, Па;

6) $K_{\mathrm{T}}$ - соотношение компонентов топлива;

7) $p_{H}$ - внешнее давление среды, Па.

Для температуры стенки управляемыми факторами являются: $\dot{m}_{\mathrm{o}}, \dot{m}_{\Gamma}, T_{\mathrm{o}}, T_{\mathrm{\Gamma}}, p_{\mathrm{K}}, K_{\mathrm{T}} ;$

$x$ - длина камеры, на которой измеряется температура;

$t$ - время работы двигателя.

Из приведенных входных факторов существенными и независимыми факторами для удельного импульса тяги являются: $\dot{m}_{\mathrm{o}}, \dot{m}_{\Gamma}, T_{\mathrm{o}}$, $T_{\Gamma}, p_{H}$, так как $K_{\mathrm{T}}=\frac{\dot{m}_{\mathrm{o}}}{\dot{m}_{\Gamma}}$, а $p_{\text {к }}$ прямо пропорционально зависит от суммарного расхода $\dot{m}_{\Sigma}=\dot{m}_{\mathrm{o}}+\dot{m}_{\text {г }}$ с следовательно, $p_{\text {к }}$ и $K_{\mathrm{T}}-$ зависимые факторы.

Внешнее давление $p_{H}$ постоянно и равно атмосферному либо вакууму $\sim 10^{-2}$ мм рт. ст. Часть двигателей испытывается на подрезанных соплах в атмосферных условиях, а часть в вакуумной камере.

Температуры компонентов топлива $T_{\text {o }}$ и $T_{\text {г }}$ при стендовых испытаниях не меняются.

Из вышеизложенного следует, что $I_{y}=f\left(\dot{m}_{\mathrm{o}}, \dot{m}_{\text {г }}\right)$. 
Существенными и независимыми факторами для температуры стенки являются: $\dot{m}_{\mathrm{o}}, \dot{m}_{\mathrm{r}}, T_{\mathrm{o}}, T_{\mathrm{r}}, x, t$. Поскольку температуры компонентов топлива на входе в двигатель не меняются, то $T_{\text {ст }}=f\left(\dot{m}_{\mathrm{o}}, \dot{m}_{\Gamma}, x, t\right)$. При испытаниях измеряется максимальная температура на сужающейся части камеры двигателя, т.е. в одной точке камеры, где тепловой поток максимален. Для упрощения регрессионной модели и уменьшения количества испытаний замер температуры стенки производится на установившемся тепловом режиме. Отсюда следует, что $T_{\text {ст }}=f\left(\dot{m}_{\mathrm{o}}, \dot{m}_{\mathrm{r}}\right)$.

Использование в регрессионной модели больше трех факторов нецелесообразно из-за неприменимости и сложности регрессионных моделей для ЖРДМТ.

Выбраны полиномиальные квазинелинейные модели и модели 2-го порядка. Квазинелинейные модели подходят для двух уровней варьирования факторов, а модели 2-го порядка - для трех. С одной стороны, эти модели отличаются простотой и требуют меньшего количества испытаний для построения адекватных зависимостей. С другой - квазинелинейная модель и полином 2-го порядка максимально точно описывают процесс в рассматриваемых диапазонах.

Применение полиномов выше 2-го порядка неоправданно, так как для их построения потребуется большее количество испытаний. Например, план полного факторного эксперимента для модели третьего порядка при трех факторах состоит из 64 опытов $\left(N=4^{3}\right)$, а с учетом повтора опытов их количество будет 128 ! Это слишком много и нецелесообразно дорого для ЖРДМТ.

Для удельного импульса наиболее оптимальные планы будут полный факторный (для двух факторов) и центральный композиционный «звездный». Для температуры стенки эти планы также подходят.

Разработаны регрессионные модели основных параметров ЖРДМТ. Общий вид моделей для удельного импульса тяги и температуры стенки выглядит следующим образом:

$$
\begin{gathered}
I_{y}=b_{0}+b_{1} \overline{\dot{m}_{\mathrm{o}}}+b_{2} \overline{\dot{m}_{\Gamma}}+b_{12} \overline{\dot{m}_{\mathrm{o}} \dot{m}_{\Gamma}}+b_{11}{\overline{\dot{m}_{\mathrm{o}}}}^{2}+b_{22}{\overline{\dot{m}_{\mathrm{\Gamma}}}}^{2}, \\
T_{\text {ст }}=b_{0}+b_{1} \overline{\dot{m}_{\mathrm{o}}}+b_{2} \overline{\dot{m}_{\mathrm{\Gamma}}}+b_{12} \overline{\dot{m}_{\mathrm{o}} \dot{m}_{\mathrm{\Gamma}}} .
\end{gathered}
$$


В табл. 1 и 2 представлены значения коэффициентов регрессии для разных двигателей.

Удельный импульс тяги

\begin{tabular}{|c|c|c|c|c|c|c|}
\hline$\overbrace{\text { Двигатель }} b_{i}, c$ & $b_{0}$ & $b_{1}$ & $b_{2}$ & $b_{12}$ & $b_{11}$ & $b_{22}$ \\
\hline ДМТ-МАИ-200М & 122,278 & $-5,83$ & 9,583 & 1,5 & - & - \\
\hline РДМТ $100^{2}$ & 405,222 & -9 & $--^{5}$ & $-5,375$ & 15,167 & $-14,083$ \\
\hline $\mathrm{S} 10^{3}$ & 291,222 & - & 3,25 & 6,875 & $-5,333$ & - \\
\hline $\mathrm{S} 400^{4}$ & 315,89 & 3,325 & - & - & - & - \\
\hline
\end{tabular}

Примечания: 1. ДМТ-МАИ-200М - ЖРДМТ, разработанный в МАИ, тягой $200 \mathrm{H}$, работающий на газообразных кислороде и метане.

2. РДМТ100 - ЖРДМТ, разработанный в ГНЦ РФ - ФГУП «Исследовательский центр им. М.В. Келдыша», тягой $100 \mathrm{H}$, работающий на газообразных кислороде и водороде.

3. S10 - ЖРДМТ, разработанный в EADS Astrium, тягой $10 \mathrm{H}$, работающий на азотном тетраоксиде и несимметричном монометилгидразине.

4. S400 - ЖРДМТ, разработанный в EADS Astrium, тягой 420 H, работающий на азотном тетраоксиде и монометилгидразине.

5. Незначимый коэффициент.

Таблица 2

Температура стенки камеры двигателя

\begin{tabular}{|c|c|c|c|c|}
\hline$\overbrace{\text { Двигатель }} b_{i}, c$ & $b_{0}$ & $b_{1}$ & $b_{2}$ & $b_{12}$ \\
\hline ДМТ-МАИ-500П & 404,5 & 11,5 & -30 & -19 \\
\hline ДМТ-МАИ-200М & 689 & -25 & 95 & 16 \\
\hline
\end{tabular}

* ДМТ-МАИ-500П - ЖРДМТ, разработанный в МАИ, тягой 200 Н, работающий на водорода пероксиде высококонцентрированном и керосине.

Для двигателей S10 и ДМТ-МАИ-200M построены графики поверхности отклика и график зависимости удельного импульса от массового расхода горючего (рис. 1-4).

В качестве фактора для построения зависимости удельного импульса выбран расход горючего, так как он оказывает линейное влияние на модель (см. рис. 1), что упрощает ее графическое представление, а расход окислителя оказывает нелинейное влияние. 


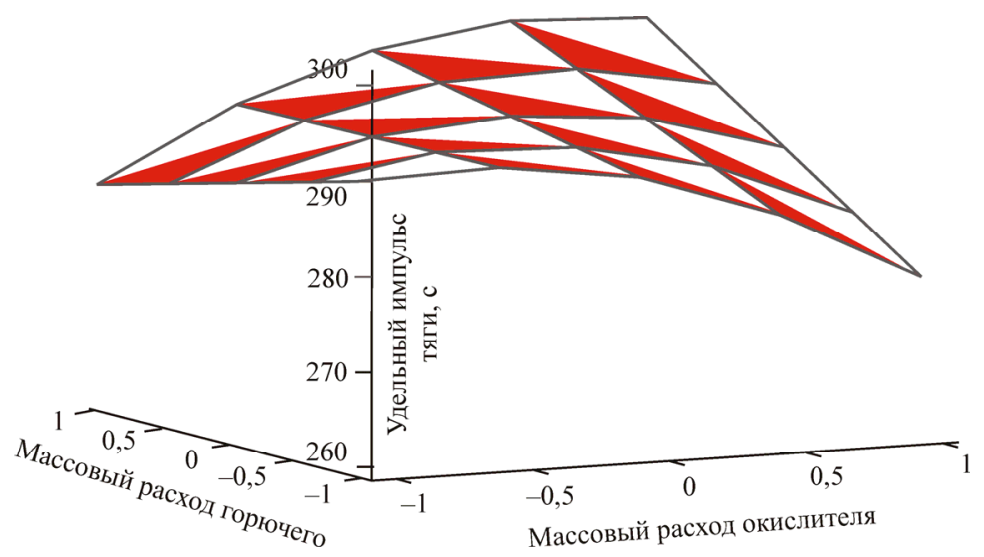

Рис. 1. График поверхности отклика удельного импульса тяги двигателя S10

Исходя из того, что расход горючего оказывает на регрессионную модель $I_{y}=291,222+3,25 \overline{\dot{m}_{\Gamma}}+6,875{\overline{\dot{m}_{\mathrm{o}}}}_{\mathrm{\Gamma}}-5,333{\overline{\dot{m}_{\mathrm{o}}}}^{2}$ линейный эффект (см. рис. 1), он взят как основной фактор при построении графика регрессионной модели при постоянном расходе окислителя 2,135 г/с (рис. 2). Он показывает, что с увеличением расхода горючего удельный импульс тяги растет. Это объясняется тем, что при увеличении суммарного расхода $\dot{m}_{\Sigma}$ растет давление в камере, что, в свою очередь, ведет к увеличению удельного импульса тяги. В данном случае влияние комплекса $R T$ незначительно из-за малой вариации факторов.

Существенным фактором для температуры стенки двигателя ДМТ-МАИ-200М является расход горючего, так как модель более чувствительна к нему (см. рис. 3).

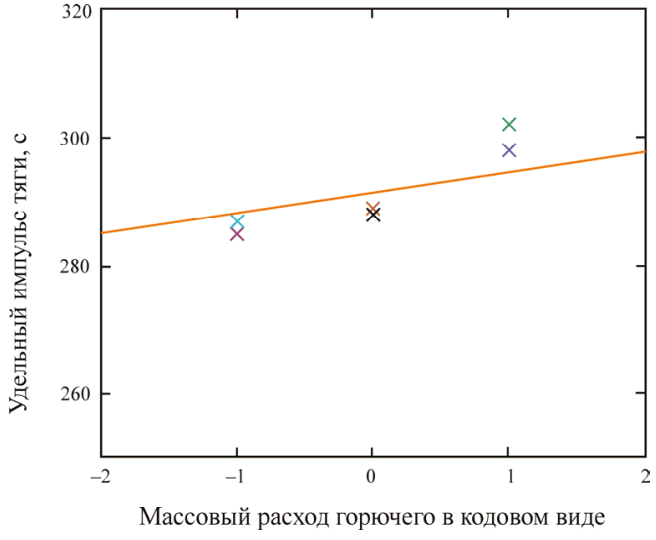

Рис. 2. Удельный импульс тяги двигателя S10 в зависимости от расхода горючего при постоянном расходе окислителя 2,135 г/с: сплошная линия - расчетная модель; $\times-$ экспериментальные точки 


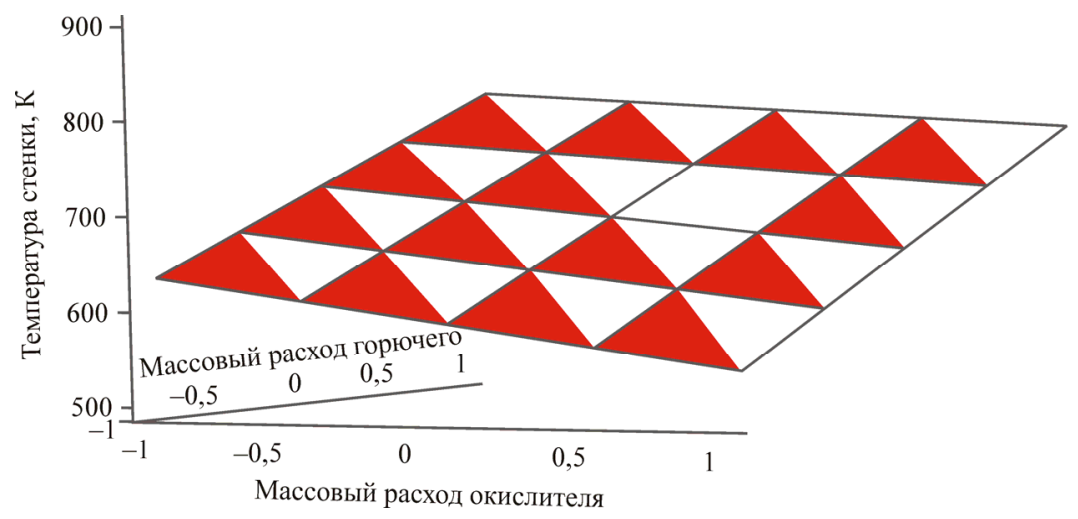

Рис. 3. График поверхности отклика температуры стенки двигателя ДМТ-МАИ-200М

Исходя из того, что график поверхности отклика температуры стенки (см. рис. 3) показывает, что регрессионная модель $T_{\text {ст }}=689-25 \overline{\dot{m}_{\mathrm{o}}}+95 \overline{\dot{m}}_{\text {г }}+16 \overline{\dot{m}_{\mathrm{o}} \dot{m}_{\text {г }}}$ более чувствительна к расходу горючего, поэтому он выбран в качестве существенного фактора. Построен график регрессионной модели при постоянном расходе окислителя 47 г/с (см. рис. 4) . Как видно из графика, с увеличением расхода горючего температура стенки растет. Это объясняется тем, что двигатель

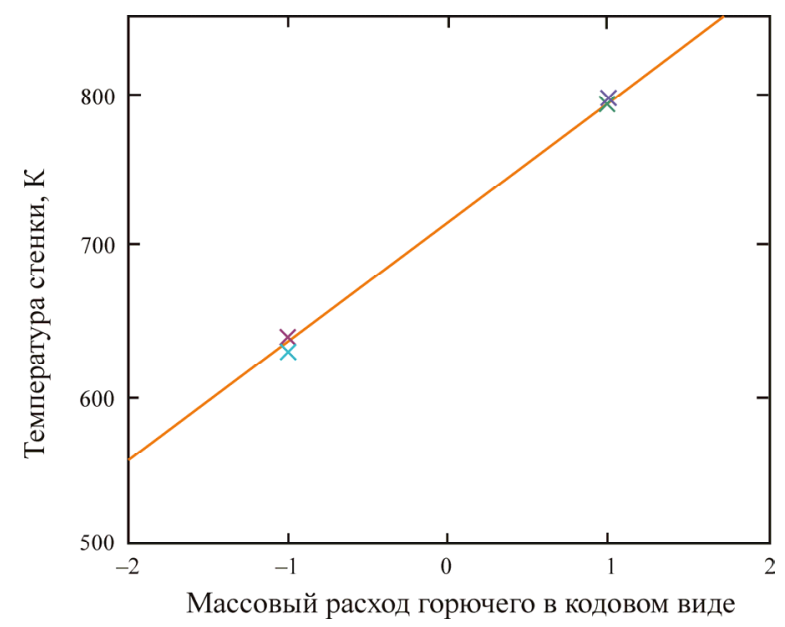

Рис. 4. Температура стенки двигателя ДМТ-МАИ$200 \mathrm{M} \mathrm{в} \mathrm{зависимости} \mathrm{от} \mathrm{расхода} \mathrm{горючего} \mathrm{при}$ постоянном расходе окислителя 47 г/с: сплошная линия - расчетная модель; $\times$ - экспериментальные точки 
работает в области после стехиометрии, т.е. при $\alpha_{\mathrm{o}}>1$, и с увеличением горючего режим работы смещается в сторону стехиометрии, следовательно, температура растет (рис. 5).

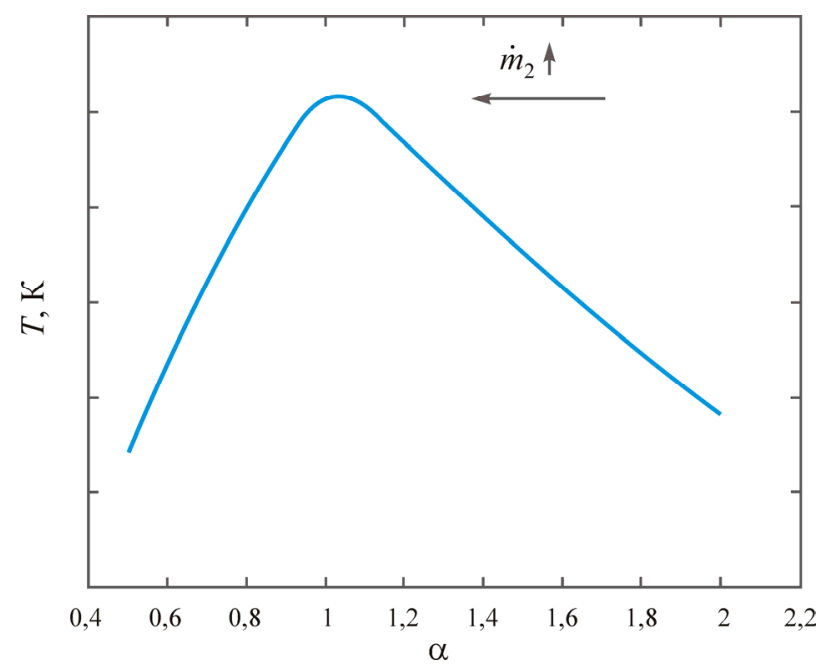

Рис. 5. Зависимость температуры стенки от коэффициента избытка окислителя

В свою очередь, при увеличении расхода окислителя и постоянном расходе горючего увеличивается суммарный расход $\dot{m}_{\Sigma}$, что ведет к увеличению давления в камере, увеличению тепловых потоков и температуры стенки.

На основании проведенного анализа различных планов экспериментов выбраны наиболее оптимальные из них. С помощью данных планов были разработаны регрессионные модели для основных параметров (удельного импульса тяги, температуры стенки камеры) ЖРДМТ. Применение оптимальных планов позволяет значительно сократить количество запусков при экспериментальной отработке двигателей, что, в свою очередь, сокращает стоимость испытаний.

\section{Библиографический список}

1. Испытания жидкостных ракетных двигателей: учеб. пособие для авиац. спец. вузов / А.Е. Жуковский, В.С. Кондрусев, В.Я. Левин, В.В. Окорочков; под ред. В.Я. Левина. - М.: Машиностроение, 1981. $199 \mathrm{c.}$ 
2. Адлер Ю.П., Маркова Е.В., Грановский Ю.В. Планирование эксперимента при поиске оптимальных условий. - 2-е изд., перераб. и доп. - М.: Наука, 1976. - 280 с.

3. Налимов В.В. Теория эксперимента. Физико-математическая библиотека инженера. - М.: Наука, 1971. - 208 с.

4. Налимов В.В., Чернова Н.А. Статистические методы планирования экстремальных экспериментов. - М.: Наука, 1965. - 340 с.

5. Адлер Ю.П. Введение в планирование эксперимента. - М.: Металлургия, 1969. - 155 с.

6. Lazic Z.R. Design of experiments in chemical engineering. Weinheim: Wiley-Vchverlag Gmbh @ Co, KGaA, 2004. - 610 p.

7. Vuchkov I.N., Lidia N., Boyadjieva N.L. Quality improvement with design of experiments: a response surface approach. Topics in safety, risk, reliability and quality. - Kluwer academic publishers, 2001. - 400 p.

8. Чуян Р.К. Методы математического моделирования двигателей летательных аппаратов. - М.: Машиностроение, 1988. - 288 с.

9. Коломенцев А.И. Учебное пособие для курсовой работы по испытаниям и обеспечению надежности жидкостных ракетных двигателей. - М.: МАИ-ПРИНТ, 2008. - 56 с.

10. Карманов В.С. Планирование эксперимента в задачах анализа данных типа времени жизни: дис. ... канд. техн. наук. - Новосибирск, 2010. - $136 \mathrm{c}$.

11. Кесаев Х.В., Трофимов Р.С. Надежность двигателей летательных аппаратов. - М.: Машиностроение, 1982. - 136 с.

12. Основы теории и расчета жидкостных ракетных двигателей: в 2 кн. Кн. 1. Учеб. для авиац. спец. вузов / А.П. Васильев, В.М. Кудрявцев, В.А. Кузнецов [и др.]; под ред. В.М. Кудрявцева. - М.: Высш. шк., 1993. - 368 с.

13. Добровольский М.В. Жидкостные ракетные двигатели. Основы проектирования / под ред. Д.А. Ягодникова. - М.: Изд-во МГТУ им. Н.Э. Баумана, 2006. -488 с.

14. Дружин А.Н. Тепловая и энергетическая эффективность до и сверхзвуковых газовых завес в ракетных двигателях малой тяги: дис. ... канд. техн. наук. - Самара, 2002. - 213 с. 


\section{References}

1. Zhukovskiy A.E., Kondrusev V.S., Levin V.Ya., Okorochkov V.V. Ispytaniya zhidkostnykh raketnykh dvigateley [Tests of liquid rocket engines]. Moscow: Mashinostroenie, 1981. 199 p.

2. Adler Yu.P., Markova E.V., Granovskiy Yu.V. Planirovanie eksperimenta pri poiske optimalnykh usloviy [Planning an experiment for searching optimum conditions]. Moscow: Nauka, 1976. 280 p.

3. Nalimov V.V. Teoriya eksperimenta. Fiziko-matematicheskaya biblioteka inzhenera [Theory of experiments. Physical and mathematical library of the engineer]. Moscow: Nauka, 1971. 208 p.

4. Nalimov V.V., Chernova N.A. Statisticheskie metody planirovaniya ekstremalnykh eksperimentov [Statistical methods of planning extreme experiments]. Moscow: Nauka, 1965. 340 p.

5. Adler Yu.P. Vvedenie v planirovanie eksperimenta [Introduction to planning an experiment]. Moscow: Metallurgiya, 1969. $155 \mathrm{p}$.

6. Lazic Z.R. Design of experiments in chemical engineering. Weinheim: Wiley-Vchverlag Gmbh @ Co, KGaA, 2004.610 p.

7. Vuchkov I.N., Lidia N., Boyadjieva N.L. Quality improvement with design of experiments: a response surface approach. Topics in safety, risk, reliability and quality. Kluwer academic publishers, 2001. $400 \mathrm{p}$.

8. Chuyan R.K. Metody matematicheskogo modelirovaniya dvigateley letatelnykh apparatov [Methods of mathematical modeling aircraft engines]. Moscow: Mashinostroenie, 1988. 288 p.

9. Kolomentsev A.I. Uchebnoe posobie dlya kursovoy raboty po ispytaniyam i obespecheniyu nadezhnosti zhidkostnykh raketnykh dvigateley [The manual for term paper on tests and ensuring reliability of liquid rocket engines]. Moscow: MAI-PRINT, 2008. 56 p.

10. Karmanov V.S. Planirovanie eksperimenta $\mathrm{v}$ zadachakh analiza dannykh tipa vremeni zhizni [Planning an experiment in problems of the analysis of like life time data]. Ph. D. thesis. Novosibirsk, 2010. 136 p.

11. Kesaev Kh.V., Trofimov R.S. Nadezhnost dvigateley letatelnykh apparatov [Reliability of aircraft engines]. Moscow: Mashinostroenie, 1982. $136 \mathrm{p}$.

12. Vasilev A.P., Kudryavtsev V.M., Kuznetsov V.A. [et. al.]. Osnovy teorii i rascheta zhidkostnykh raketnykh dvigateley [Basics of the theory and calculation of liquid rocket engines]. Moscow: Vysshaia shkola, 1993. $368 \mathrm{p}$. 
13. Dobrovolskiy M.V. Zhidkostnye raketnye dvigateli. Osnovy proektirovaniya [Liquid rocket engines. Design basis]. Moscovskiy gosudarstvennyy tekhnicheskiy universitet imeni N.E. Baumana, 2006. 488 p.

14. Druzhin A.N. Teplovaya i energeticheskaya effektivnost do i sverkhzvukovykh gazovykh zaves $\mathrm{v}$ raketnykh dvigatelyakh maloy tyagi [Thermal and power efficiency of subsonic and supersonic gas veils in low-thrust rocket engines]. Ph. D. thesis. Samara, 2002. 213 p.

\section{Об авторах}

Коломенцев Александр Иванович (Москва, Россия) - кандидат технических наук, профессор Московского авиационного института (национального исследовательского университета) (125993, г. Москва, Волоколамское ш., д. 4, e-mail: kaf202@mai.ru).

Хохлов Алексей Николаевич (Москва, Россия) - кандидат технических наук, заместитель начальника отдела Центрального научноисследовательского института машиностроения (141070, г. Королев, ул. Пионерская, д. 4, e-mail: alexey.hohlov.86@gmail.com).

\section{About the authors}

Alexander I. Kolomentsev (Moscow, Russian Federation) - Ph. D. in Technical Sciences, Professor, Moscow Aviation Institute (National Research University) (4, Volokolamskoe shosse, Moscow, 125993, Russian Federation, e-mail: kaf202@mai.ru).

Alexey N. Khokhlov (Moscow, Russian Federation) - Ph. D. in Technical Sciences, Deputy Head of a Department, Central Research Institute of Machine Building (4, Pionerskaya st., Korolev, 141070, Russian Federation, e-mail: alexey.hohlov.86@gmail.com).

Получено 03.10.2016 